\title{
Transient Formation of Super-Explosives under High Pressure for Fast Ignition*
}

\author{
Friedwardt Winterberg \\ University of Nevada, Reno, NV 89523, USA \\ Reprint requests to Prof. F. W.; E-mail: winterbe@unr.edu \\ Z. Naturforsch. 63a, 35-41 (2008); received July 20, 2007
}

\begin{abstract}
Dense matter, if put under high pressure, can undergo a transformation from an atomic to a molecular configuration, where the electron orbits go into lower energy levels. If the rise in pressure is very sudden, for example by a strong shock wave, the electrons change their orbits rapidly under the emission of photons, which for more than 100 Mbar can reach $\mathrm{keV}$ energies. With the opacity of dense matter going in proportion to the density, the photons can be efficiently released from the surface of the compressed matter by a rarefaction wave. The so produced X-ray photons can be used for the fast ignition of a thermonuclear target.

The proposed mechanism may be also responsible for the large keV X-ray bursts observed in exploding wire arrays, which can not be explained by conversion of kinetic into thermal energy.
\end{abstract}

Key words: keV Chemistry; High Pressure; Fast Ignition.

\section{Introduction}

Ballotechnics are combustible mixtures of chemically reacting powders which, unlike high explosives, do not produce volatile gases, but mainly heat [1-5]. This means that the reaction products consist of heavy elements with low thermal velocities. Related to ballotechnics is an iron oxide/aluminium mixture, known as thermite, with the reaction products being liquid iron and aluminium oxide. It releases as much energy per mass as the best high explosives. While thermite just needs a fuse for ignition, other ballotechnics also need a high pressure comparable to the energy density of chemical reactions, or of the order $10^{11}$ $10^{12} \mathrm{dyn} / \mathrm{cm}^{2}$, or less than $1 \mathrm{Mbar}$. The energy released in both high explosives and ballotechnics is through a change in electron orbits, amounting to not more than a few electron volts.

A very different situation is likely to occur if the pressure is much larger, where atomic nuclei get much closer to each other, forming a deeper potential well for the electrons. By falling down into the well, the electrons emit photons which, if the well is deep enough, can be quite energetic. In a simplified way, this can be seen as follows: the energy of an electron in the groundstate of a nucleus with the charge $Z e$ is

$$
E_{1}=-13.6 Z^{2}[\mathrm{eV}] .
$$

* Paper B06 15, Bull. Am. Phys. Soc. 52, 33 (2007).
With the inclusion of all the $Z$ electrons surrounding the nucleus of charge $Z e$, the energy is

$$
E_{1}^{*} \approx-13.6 Z^{2.42}[\mathrm{eV}]
$$

with the outer electrons less strongly bound to the nucleus.

Now, assume that two nuclei are so strongly pushed together that they act like one nucleus with the charge $2 Z e$ onto the $2 Z$ electrons surrounding the $2 Z e$ charge. In this case, the energy for the innermost electron is

$$
E_{2}=-13.6(2 Z)^{2}[\mathrm{eV}],
$$

or if the outer electrons are taken into account,

$$
E_{2}^{*}=-13.6(2 Z)^{2.42}[\mathrm{eV}] .
$$

For the difference one obtains

$$
\begin{aligned}
\delta E & =E_{1}^{*}-E_{2}^{*} \\
& =13.6 Z^{2.42}\left(2^{2.42}-1\right) \approx 58.5 Z^{2.42}[\mathrm{eV}] .
\end{aligned}
$$

Using the example $Z=10$, which is a neon nucleus, one obtains $\delta E \approx 15 \mathrm{keV}$. Of course, it would require a very high pressure to push two neon atoms that close to each other, but this example makes it plausible that smaller pressures exerted on heavier nuclei with many more electrons may result in a substantial lowering of the potential well for their electrons. 
If the attainable values for $\delta E$ are in the $\mathrm{keV}$ energy range, with the photon absorption going down with increasing photon energy, it raises the question if the photons released under very high pressures can be used for the fast ignition of thermonuclear targets.

\section{Attainment of 100 Mbar Pressures}

We are aiming at pressure $p \approx 100 \mathrm{Mbar}=$ $10^{14} \mathrm{dyn} / \mathrm{cm}^{2}$, which can be reached with existing technology in sufficiently large volumes.

There are three possibilities: (i) bombardment of a solid target with an intense relativistic electron- or ion beam; (ii) hypervelocity impact; (iii) bombardment of a solid target with beams or by hypervelocity impact, followed by a convergent shock wave.

To (i): This possibility was considered by Kidder [6] who computed a pressure of $50 \mathrm{Mbar}$, if an iron plate is bombarded with a $1 \mathrm{MJ}-10 \mathrm{MeV}-10^{6}$ A relativistic electron beam, focused down to an area of $0.1 \mathrm{~cm}^{2}$. A $2 \mathrm{MJ}$ beam would produce $100 \mathrm{Mbar}$. Instead of using an intense relativistic electron beam, one may use an intense ion beam, which can be produced by the same high voltage technique, replacing the electron beam diode by a magnetically insulate diode [7].

To (ii): A projectile with the density $\rho \approx 20 \mathrm{~g} / \mathrm{cm}^{3}$, accelerated to a velocity of $v=30 \mathrm{~km} / \mathrm{s}$ would, upon impact, produce a pressure of $p \approx 100$ Mbar. The acceleration of the projectile to this velocity can be done by a magnetic traveling wave accelerator $[8,9]$.

To (iii): If, upon impact of either a particle beam or projectile, the pressure is less than $100 \mathrm{Mbar}$, for example only of the order 10 Mbar, but over a larger area, a ten-fold increase in the pressure over a smaller area is possible by launching a convergent shock wave from the larger onto a smaller area. According to Guderley [10], the rise in pressure in a convergent spherical shock wave goes as $r^{-0.9}$, which means that $100 \mathrm{Mbar}$ could be reached by a ten-fold reduction in the radius of the convergent shock wave.

While it is difficult to reach $30 \mathrm{~km} / \mathrm{s}$ with a traveling magnetic wave accelerator, it is easy to reach a velocity of $10 \mathrm{~km} / \mathrm{s}$ with a two-stage light gas gun.

\section{Solid Matter under a Pressure of 100 Mbar}

At a pressure of $100 \mathrm{Mbar}$, we may in the equation of state for dense matter, $p / p_{0}=\left(n / n_{0}\right)^{\gamma}$, set $\gamma=3$ and $p_{0}=10^{11} \mathrm{dyn} / \mathrm{cm}^{2}, p_{0}$ being the Fermi pressure of a solid at the atomic number density $n_{0}$, with $n$ being the atomic number density at the elevated pressure $p>p_{0}$. With $d=n^{-1 / 3}$, where $d$ is the lattice constant, one has

$$
d / d_{0}=\left(p_{0} / p\right)^{1 / 9} \text {. }
$$

For $p=10^{14} \mathrm{dyn} / \mathrm{cm}^{2}$, one finds that $d / d_{0} \sim 1 / 2$. Such a lowering of the inneratomic distance is sufficient for the formation of molecular states.

\section{Emission of X-Rays under the Sudden Application of a High Pressure Pulse}

Calculations done by Müller, Rafelski, and Greiner [11] (see Appendix A) show that for the molecular states ${ }^{35} \mathrm{Br}-{ }^{35} \mathrm{Br},{ }^{53} \mathrm{I}-{ }^{79} \mathrm{Au}$, and ${ }^{92} \mathrm{U}-{ }^{92} \mathrm{U}$, a two-fold lowering of the distance of separation leads to a lowering of the electron orbit energy eigenvalues by $\sim 0.35 \mathrm{keV}, 1.4 \mathrm{keV}$, and $10 \mathrm{keV}$, respectively. These results confirm the rough, order of magnitude, estimates made in the introduction.

The effect a pressure has on a change in these quasi-molecular configurations is illustrated in Fig. 1, showing a pressure-lattice distance diagram. In this diagram, the upper curve and lower curves are adiabats, where with a rise in the pressure, the lattice distance decreases. But because the electrons can fall from the higher into the lower adiabat of the quasi-molecular configuration, the electrons release the excess energy by X-rays. These X-rays have a short wavelength, and their absorption is determined by an opacity given by [12]

$$
\kappa=\text { const. } \rho T^{-3.5} \text {. }
$$

This means that with the opacity going in proportion of the density $\rho$, the X-rays can be released from the rarefaction wave, starting from the surface of the matter, which is under high pressure.

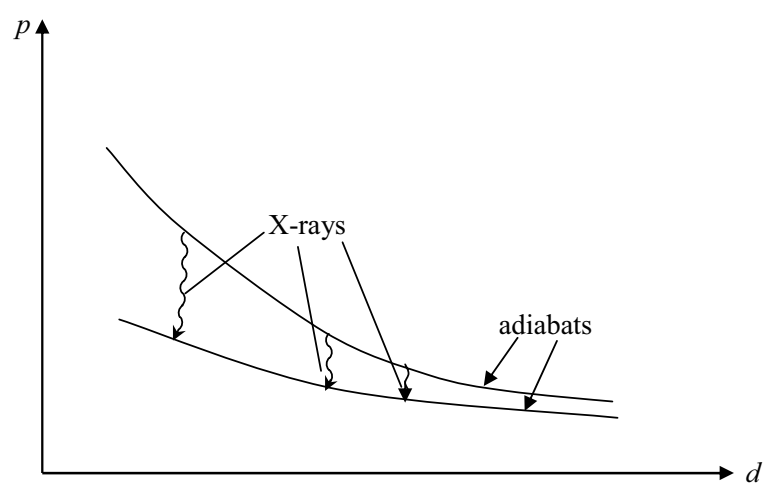

Fig. 1. Pressure-lattice distance diagram for the upper atomic and lower molecular adiabat. 


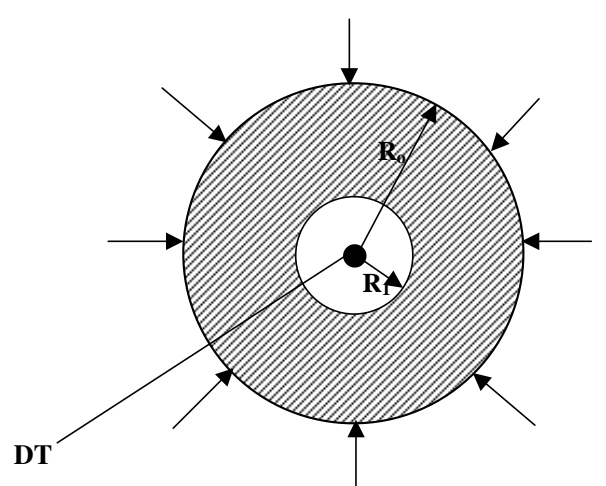

Fig. 2. Inertial confinement fast ignition configuration.

For the fast ignition of small thermonuclear targets, two methods have been proposed. In the first, a highly compressed DT (deuterium-tritium) target is ignited with a $\sim 100 \mathrm{~kJ}$ petawatt laser pulse, creating a hot spot in the target, which launches a thermonuclear detonation wave [13]. Because a petawatt laser with this kind of energy output would be very expensive, an alternative proposal was made suggesting that the same laser which compresses the target could, at the same time, accelerate a foil to a velocity of $\sim 10^{8} \mathrm{~cm} / \mathrm{s}$, which upon impact on the highly compressed target would do the same [14].

But here we may consider a third possibility which makes use of Guderley's convergent shock wave solution [10]. It is the fast ignition method presumably used in large thermonuclear explosive devices, where a hot spot is created in the centre of convergence.

One may consider the following scenario illustrated in Figure 2. A convergent shock wave, launched at the radius $R=R_{0}$ into a spherical shell of outer and inner radius $R_{0}$ and $R_{1}$, reaches near the radius $R=R_{1}$ a pressure of $100 \mathrm{Mbar}$. After the inward moving convergent shock wave has reached the radius $R=R_{1}$, an outward moving rarefaction wave is launched from the same radius $R=R_{1}$, from which an intense burst of $\mathrm{X}$-rays is emitted. One can then place a thermonuclear target inside the cavity of the radius $R=R_{1}$, with the target bombarded, imploded, and ignited by the $\mathrm{X}$-ray pulse.

The X-rays get their energy from the work done by the pressure force which pushes the atoms towards each other. One can distinguish three cases. In the first case, the compressive collision between the atoms is completely elastic. For a system of many atoms, this corresponds to an isentropic equation of state. There no X-rays will be released. In the second case, the col- lision is inelastic, but not completely inelastic. In this case the kinetic energy lost in the collision is emitted as $\mathrm{X}$-rays. Finally, in the third case, where the collision is completely inelastic, the maximum amount of energy is released as X-rays. In this case, the two atoms stick together forming a bound state. This happens, for example, in the formation of diamond from graphite, and it might happen in the formation of metallic hydrogen. But in both of these cases, more than two atoms stick to each other.

If the material to be compressed is made up of different atoms, as in the quasi-molecular ${ }^{53} \mathrm{I}^{79} \mathrm{Au}$ system, the two components must form an alloy, and if this is not possible, they must be a mixture of nano-particle powders. Apart from a two-component system, mixtures of more than two species of atoms are, of course, also possible. There the predicted effect may be even larger.

\section{X-Ray Flux Released by High Pressure}

As shown in Fig. 1, the emission of the X-rays is accompanied by a transition from the upper to the lower adiabat and by a fall of the pressure. If the change in pressure is large, whereby the pressure in the upper adiabat is large compared to the pressure in the lower adiabat, the X-ray energy flux is given by the photon diffusion equation

$$
\phi=-\frac{\lambda c}{3} \nabla w,
$$

where $w$ is the work done per unit volume to compress the material, where $w=p /(\gamma-1)$.

For $\gamma=3$, one has $w=p / 2$, whereby (8) becomes

$$
\phi=-\frac{\lambda c}{6} \nabla p .
$$

Assuming that the pressure e-folds over the same length as the photon mean free path, one has

$$
\phi \sim(c / 6) p .
$$

For the example $p=100 \mathrm{Mbar}=10^{14} \mathrm{dyn} / \mathrm{cm}^{2}$ one finds that $\phi \sim 5 \cdot 10^{23} \mathrm{erg} /\left(\mathrm{cm}^{2} \mathrm{~s}\right)=5 \cdot 10^{16} \mathrm{~W} / \mathrm{cm}^{2}$.

Placing a DT capsule with an ablator of critical density $\rho$ inside an X-ray-generating shell, the ablation pressure on the capsule is given by (for a simplified derivation see Appendix B)

$$
p=(4 / 15)^{1 / 3} \phi^{2 / 3} \rho^{1 / 3} .
$$


For the example $\phi \sim 5 \cdot 10^{23} \mathrm{erg} /\left(\mathrm{cm}^{2} \mathrm{~s}\right)=5$. $10^{16} \mathrm{~W} / \mathrm{cm}^{2}, \rho \approx 1 \mathrm{~g} / \mathrm{cm}^{3}$, one finds $p \approx 4$. $10^{15} \mathrm{dyn} / \mathrm{cm}^{2}$, and for the implosion velocity $v=$ $\sqrt{\gamma p / \rho}(\gamma=5 / 3)$, one finds $v \approx 10^{8} \mathrm{~cm} / \mathrm{s}$, sufficient for thermonuclear ignition and burn.

\section{How to Find and Prepare the Best Super-Explosive}

The most energetic, X-ray-emitting super-explosive can be found by searching through the experimentally obtained data for all combinations of ion-ion collisions, and determining which of those emit the largest amount of X-rays.

If the conjectured super-explosive consists of just one element, as is the case for the ${ }^{35} \mathrm{Br}^{-}{ }^{35} \mathrm{Br}$ reaction or the ${ }^{92} \mathrm{U}-{ }^{92} \mathrm{U}$ reaction, no special preparation for the super-explosive is needed. But as the example of $\mathrm{Al}-\mathrm{FeO}$ thermite reaction shows, reactions with different atoms can release a much larger amount of energy compared to other chemical reactions. For conjectured super-explosives this means that they have to be prepared as homogeneous mixtures of nano-particle powders, bringing the reacting atoms as close together as possible.

\section{Possible Connection to the Release of Intense $X$-Ray Bursts in Electric Pulse Power-Driven Exploding Wire Arrays}

The outstanding unresolved question in the release of intense X-ray bursts in electric pulse power-driven imploding wire arrays is the unexpected occurrence of a large contribution in the keV energy range. It can not be explained by a simple kinetic into thermal energy conversion model, which predicts a black body radiation spectrum in the sub-keV range $[15,16]$. Models that account for this discrepancy are 1) enhanced magnetic energy dissipation through microturbulenceincreased electrical resistivity [17], and 2) the generation of bremsstrahlung by the formation of electron beams [18].

To explore the possibility whether or not these X-ray bursts can be explained by the proposed mechanism, we take as an example a thin wire with a radius $r=$ $2 \cdot 10^{-3} \mathrm{~cm}$ over which a current of $I=10^{6} \mathrm{~A}$ is flowing. The magnetic field at the surface of the wire is $B=0.2 I / r=10^{8} \mathrm{G}$, with a magnetic pressure $B^{2} / 8 \pi=$ $4 \cdot 10^{14} \mathrm{dyn} / \mathrm{cm}^{2}$, acting on the wire, sufficiently large for the conjectured release of keV X-rays by $100 \mathrm{Mbar}$ pressures.

\section{Conclusion}

The ignition of thermonuclear micro-explosions requires an energy of more than $1 \mathrm{MJ}\left(10^{13} \mathrm{erg}\right)$, to be delivered onto an area less than $1 \mathrm{~cm}^{2}$ in less than $10^{-8} \mathrm{~s}$. This energy is really not that large, but difficult to deliver in the short time by laser- or particle beams. Even at the required beam intensity, the particle number density in the beam is much smaller than the particle number density of the solid state. The only kind of beam, where the number density is comparable, is in impact fusion, where the beam is a single solid particle (marco-particle), accelerated to high velocities. There the energy flux density is $\phi=(1 / 2) \rho v^{3}$, where $\rho$ is the density of the macro-particle and $v$ its velocity. For the example $\rho=20 \mathrm{~g} / \mathrm{cm}^{3}$, a velocity of $v=50 \mathrm{~km} / \mathrm{s}$ is needed to reach $\phi=100 \mathrm{TW}\left(10^{21} \mathrm{erg} / \mathrm{s}\right)$. This velocity is less than $v=200 \mathrm{~km} / \mathrm{s}$ needed for impact fusion, but sufficient if the impact energy can be converted into a burst of radiation to implode and ignite a thermonuclear target. The conversion of the kinetic impact energy into heat, with the energy emitted as thermal radiation, is not very efficient if a large fraction of the heat is trapped inside the solid. This is different if the energy stored in the pressure is directly converted into $\mathrm{X}$-rays, having a large range.

If realizable, the concept presented here opens new avenues for the achievement of nuclear fusion by inertial confinement. To reach a pressure of $100 \mathrm{Mbar}$, one can use intense relativistic electron beams, which are less expensive by orders of magnitude than lasers. In comparison to soft X-rays emitted by exploding wire arrays, the concept does not need replaceable transmission lines, because intense relativistic electron beams can with ease be transported through a tenuous background gas. The concept also sheds new light on fusion through the acceleration of macro-particles, because if coupled with the generation of X-rays by the impact pressure of the macro-particles, smaller velocities suffice than the velocities otherwise required for impact fusion.

\section{Appendix A: The Quantum Mechanical Eigenval- ues of Two-Atom Molecule Two-Centre Configura- tions}

This is the extension of the Heitler-London theory for the chemical bonding of two hydrogen atoms to 


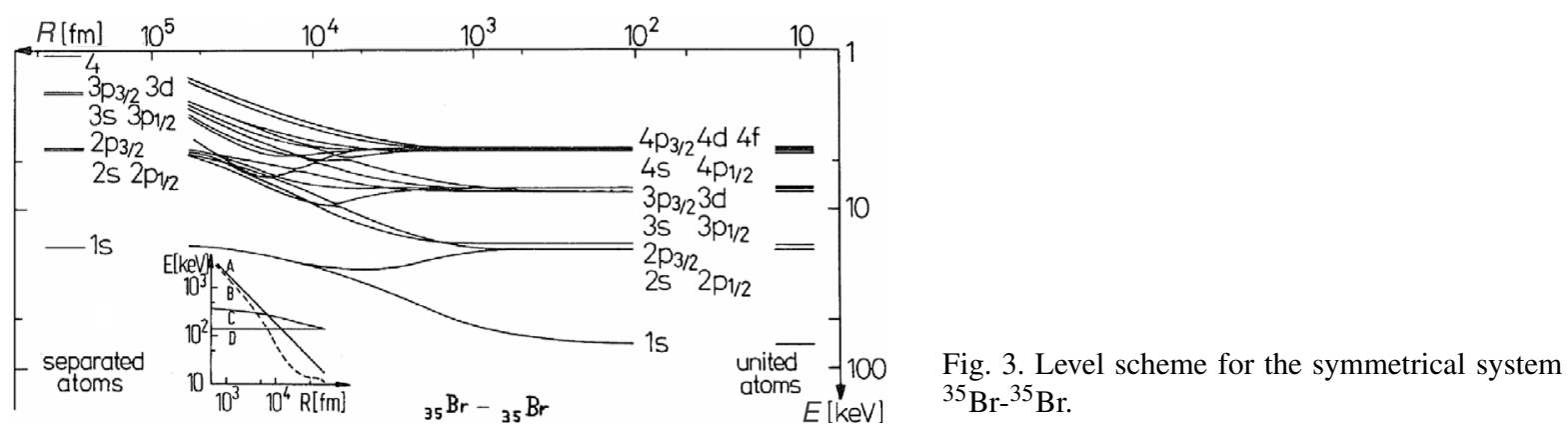

heavier elements. It is fortunate that this problem has already been solved to predict the outcome of heavy atom collisions, and one can simply use the results obtained [11]. Because in the collision of the heaviest atoms, the effective two-centre electric charge can exceed the critical value $Z_{\text {crit }}=137$, above which vacuum breakdown begins to start. One has here to use the twocentre Dirac equation for an electron of mass $M$ in the field of two electric charges located at $-\boldsymbol{R}$ and $\boldsymbol{R}$ :

$$
\left[c \boldsymbol{\alpha} \cdot \boldsymbol{p}+\beta M c^{2}-E+V_{1}(\boldsymbol{r}-\boldsymbol{R})+V_{2}(\boldsymbol{r}+\boldsymbol{R})\right] \Psi=0 .
$$

Introducing prolate spheroidal coordinates $\xi, \eta, \varphi$, one has with the $z$-axis going from $-\boldsymbol{R}$ to $\boldsymbol{R}$

$$
\begin{aligned}
& x=R\left[\left(\xi^{2}-1\right)\left(1-\eta^{2}\right)\right]^{1 / 2} \cos \varphi, \\
& y=R\left[\left(\xi^{2}-1\right)\left(1-\eta^{2}\right)\right]^{1 / 2} \sin \varphi, \\
& z=R \xi \eta .
\end{aligned}
$$

With the angular momentum $m+1 / 2$ around the $z$ axis, the $\varphi$ dependence in (A.1) can be separated setting

$$
\Psi(\boldsymbol{r})=\left(\begin{array}{llll}
\mathrm{e}^{\mathrm{i} m \varphi} & & \\
& \mathrm{e}^{\mathrm{i}(m+1) \varphi} & & \\
& & \mathrm{ie}^{\mathrm{i} m \varphi} & \\
& & \mathrm{ie}^{\mathrm{i}(m+1) \varphi}
\end{array}\right) \Psi^{\prime}(\xi, \eta)
$$

resulting in

$$
\begin{gathered}
{\left[\frac{\hbar c}{R\left(\xi^{2}-\eta^{2}\right)}\left(\begin{array}{cccc}
0 & 0 & \pi_{z} & \pi^{-} \\
0 & 0 & \pi^{+} & -\pi_{z} \\
\pi_{z} & -\pi^{-} & 0 & 0 \\
-\pi^{+} & \pi_{z} & 0 & 0
\end{array}\right)+\beta M c^{2}\right.} \\
\left.-E+V_{1}(\xi+\eta)+V_{2}(\xi-\eta)\right] \Psi^{\prime}(\xi, \eta)=0,
\end{gathered}
$$

where

$$
\begin{aligned}
& \pi^{+}=W\left(\xi \frac{\partial}{\partial \xi}-\eta \frac{\partial}{\partial \eta}\right)-\frac{m}{W}\left(\xi^{2}-\eta^{2}\right), \\
& \pi^{-}=W\left(\xi \frac{\partial}{\partial \xi}-\eta \frac{\partial}{\partial \eta}\right)+\frac{m+1}{W}\left(\xi^{2}-\eta^{2}\right), \\
& \pi_{z}=\eta\left(\xi^{2}-1\right) \frac{\partial}{\partial \xi}+\xi\left(1-\eta^{2}\right) \frac{\partial}{\partial \eta} \\
& W=\left[\left(\xi^{2}-1\right)\left(1-\eta^{2}\right)\right]^{1 / 2} .
\end{aligned}
$$

Equation (A.4) is separable in a $\xi$ and $\eta$ dependence setting

$$
\begin{aligned}
\Psi_{n l s}^{m}(\xi, \eta)= & \left(\xi^{2}-1\right)^{\left(m+\varepsilon_{s}\right) / 2} \exp \left(-\frac{\xi-1}{a}\right) \\
& \cdot L_{n}^{m+\varepsilon_{s}}\left(\frac{\xi-1}{a}\right) P_{l}^{m+\varepsilon_{s}}(\eta) \chi_{s},
\end{aligned}
$$

where $\mathrm{L}_{i}^{\alpha}$ and $\mathrm{P}_{i}^{\alpha}$ are the associated Laguerre and Legendre polynomials. In (A.6), $a$ is a scaling factor, not affecting the energy eigenvalue $E$. Furthermore, $\chi_{s}$ are the unit spin vectors, $\varepsilon_{s}=0$ for $s$ odd and $\varepsilon_{s}=1$ for $s$ even. Inserting (A.6) into (A.4), one obtains the energy eigenvalues. The results for three configurations, ${ }^{35} \mathrm{Br}-{ }^{35} \mathrm{Br},{ }^{53} \mathrm{I}^{79} \mathrm{Au}$, and ${ }^{92} \mathrm{U}-{ }^{92} \mathrm{U}$ are displayed in Figs. 3-5, respectively. As noticed by Müller, Rafelski, and Greiner [11], the energy eigenvalues exhibit a "run way" towards distances of separation of the order $10^{3} \mathrm{fm}$.

If the two atoms just touch each other without the application of an external pressure, one can approximately set for their distance of separation $1 \AA=$ $10^{5} \mathrm{fm}$. Under the high pressure of $10^{14} \mathrm{dyn} / \mathrm{cm}^{2}$, their mutual distance of separation is reduced from $10^{5} \mathrm{fm}$ down to $5 \cdot 10^{4} \mathrm{fm}$. For the uppermost energy levels, this implies a lowering of the energy eigenvalues by $\sim 0.35 \mathrm{keV}$ for the ${ }^{35} \mathrm{Br}^{-35} \mathrm{Br}$ system, by $\sim 1.4 \mathrm{keV}$ 


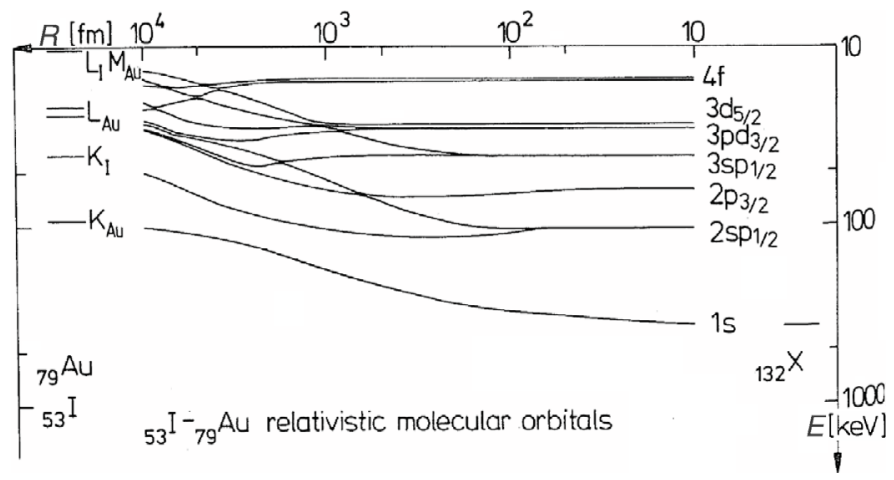

Fig. 4. Level scheme for the asymmetrical system

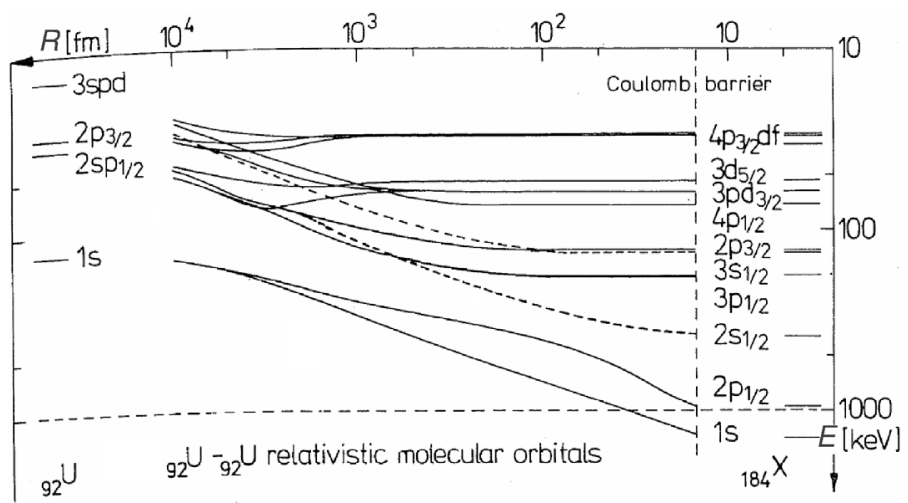

Fig. 5. The ${ }^{92} \mathrm{U}^{92} \mathrm{U}$ system.

for the ${ }^{53} \mathrm{I}_{-}{ }^{79} \mathrm{Au}$ system, and by $10 \mathrm{keV}$ for the ${ }^{92} \mathrm{U}-{ }^{92} \mathrm{U}$ system. Therefore, if the compression is very abrupt, the emission of keV X-rays seems possible.

\section{Appendix B: The Ablation Pressure}

A simplified derivation of the ablation pressure is obtained from the two rocket equations for thrust $T$ and power $\mathrm{d} E / \mathrm{d} t$ ( $v$, ablation velocity):

$$
\begin{aligned}
& T=v \frac{\mathrm{d} m}{\mathrm{~d} t}, \\
& \frac{\mathrm{d} E}{\mathrm{~d} t}=\frac{v^{2}}{2} \frac{\mathrm{d} m}{\mathrm{~d} t} .
\end{aligned}
$$

With the ablation pressure equal the thrust per unit area, and likewise the radiation intensity $\phi$ equal the power per unit area, one obtains

$$
p=2 \frac{\phi}{v}
$$

This expression is only correct if all the ablated material leaves the surface in a perpendicular direction. In reality, the ablated material is isotropically ejected into the solid angle $2 \pi$. A jet emitted per unit area from the surface into the direction $\theta$ of a spherical coordinate system has the cross-section $\cos \theta$, and only the fraction $\cos \theta$ of its recoil is transmitted to the surface. With the element of the solid angle $\mathrm{d} \omega=2 \pi \sin \theta \mathrm{d} \theta$ we have to integrate over all directions to obtain the pressure per unit solid angle:

$$
p=\frac{2 \phi}{v} \frac{1}{2 \pi} \int_{0}^{\pi / 2}\left(\cos ^{2} \theta\right) \mathrm{d} \omega \mathrm{d} \theta=\frac{2}{3} \frac{\phi}{v} .
$$

Setting $v$ equal the velocity of sound, $v=\sqrt{\gamma p / \rho}$, one obtains, for $\gamma=5 / 3, v=\sqrt{5 / 3} \sqrt{p / \rho}$, and hence from (B.4)

$$
p=(4 / 15)^{1 / 3} \phi^{2 / 3} \rho^{1 / 3} .
$$

\section{Acknowledgement}

The idea presented in this paper, first considered in 1974, was stimulated by the paper of B. Müller, J. Rafelski, and W. Greiner, and I acknowledge the very useful conversation I had at that time with Dr. Rafelski in Frankfurt, Germany. 
[1] R. A. Graham, in: Shock Waves in Condensed Matter, Monterey, California, 20-23 July 1987, pp. 11-18.

[2] M. B. Boslough, A Thermochemical Model for ShockInduced Chemical Reactions in Porous Solids: Analogs and Contrast to Detonation, in: International Symposium on Detonation, 28 Aug. - 1 Sept. 1989, Portland, Oregon, USA.

[3] N.S. Enikolopyan, A. A. Khzardzhyan, E.E. Gasparyan, and V. B. Vasil'eva, Acad. Nauk. USSR, Proc. Phys. Chem. 294, 567 (1987).

[4] M. B. Boslough and R. A. Graham, Chem. Phys. Lett. 121, 446 (1985).

[5] X. Xu and N. N. Thadhani, J. Appl. Phys. 96, 2000 (2004).

[6] R. E. Kidder, in: Physics of High Energy Density, Academic Press, New York 1971, pp. 306-352.

[7] F. Winterberg, in: Physics of High Energy Density, Academic Press, New York 1971, pp. 397 - 401.

[8] C. Maisonnier, Nuovo Cimento 426, 332 (1966).

[9] F. Winterberg, J. Nucl. Energy C 8, 541 (1966).

[10] G. Guderley, Luftfahrtforschung 19, 302 (1942).

[11] B. Müller, J. Rafelski, and W. Greiner, Phys. Lett. 47B, 5 (1973).

[12] M. Schwarzschild, Structure and Evolution of the
Stars, Princeton University Press, Princeton, New Jersey 1958 , pp. $62-73$.

[13] M. Tabak, J. Hammer, M. E. Glinsky, W. L. Kruer, S. C. Wilks, J. Woodworth, E.M. Campbell, M.D. Perry, and R. J. Mason, Phys. Plasmas 1, 1626 (1994).

[14] M. Murakami and H. Nagatomo, Nuclear Instrum. Methods Phys. Res. A 544, 67 (2005).

[15] T. W. L. Sanford, T. J. Nash, R. C. Mock, R. B. Spielman, K. W. Struve, J. H. Hammer, J. S. De Groot, K. G. Whitney, and J. P. Apruzese, Phys. Plasmas 69, 02188 (1997).

[16] C. Deeney, M. R. Douglas, R. B. Spielman, T. J. Nash, D. L. Peterson, P. L'Eplattenier, G. A. Chandler, J. F. Seamen, and K. W. Struve, Phys. Rev. Lett. 81, 4883 (1998).

[17] L. I. Rudakov, A. L. Velikovich, J. Davis, J. W. Thornhill, J. L. Giuliani, Jr., and C. Deeney, Phys. Rev. Lett. 84, 3326 (2000).

[18] V. L. Kantsyrev, L. I. Rudakov, A. S. Safronova, D. A. Fedin, V. V. Ivanov, A.L. Velikovich, A. A. Esualov, A. S. Chuvatin, K. Williamson, N.D. Ouart, V. Nalajala, G. Osborne, I. Shrestha, M. F. Yilmaz, S. Pokala, P. J. Laca, and T.E. Cowan, IEEE Trans. Plasma Sci. 34, 2295 (2006). 